\title{
Aerobic Capacity Index and Its Information Management Data Structure
}

\author{
Zhu Li \\ College of Physical Education, Dalian University, Dalian, 116622, China \\ 48269162@qq.com
}

Keywords: aerobic capacity; index system; information management; data structure

\begin{abstract}
Aerobic capacity mainly uses the physiological and biochemical indexes, timely understands athletes' body function condition, which has important significance for the selected material of athletes, medical supervision, reasonable arrangements for training plans, judging whether fatigue occurs, effectively mining the potential of human body movement, improving the competitive ability and so on. The research content of this paper mainly includes two aspects, one is that the analysis of nine aerobic metabolism indexes that the "Direct maximal oxygen uptake, Relative maximal oxygen uptake, $\mathrm{VO}_{2}$ max pleteau duration, PWC170, Maximal lactate steady state, Lactate anaerobic threshold, Ventilation anaerobic threshold, Break point anaerobic threshold, Respiratory quotient", the second is that the data storage logic structure is constructed which consists of three tables of "Athlete information table, Sampling information table, Index value information table" based on MySQL database management system, providing support for the system development.
\end{abstract}

\section{Introduction}

Aerobic exercise refers to the aerobic metabolism is taken as a form of energy supply during exercise, characterized by low intensity, rhythmic, without interruption and long duration. Doing aerobic exercise, on the one hand, can increase the body's absorption, the ability of transporting and using oxygen; on the other hand can promote the body's blood circulation, improve microcirculation and internal environment, enhance metabolism, improve the physiological function of the important organs such as brain and heart lung. The energy supply of aerobic exercise mainly comes from carbohydrates and fats, if the obese can persist in aerobic exercise, the excess calories and fat can be effectively consumed in the body, which can lose weight, and can play a role of lowering blood pressure, blood lipid, blood sugar and improving body immunity, also beneficial to prevent arteriosclerosis, hypertension, coronary heart disease, stroke, diabetes, cancer and other diseases. Aerobic capacity mainly uses the physiological and biochemical indexes, timely and accurately understands athletes' body function condition, which has important significance for the selected material, medical supervision, reasonable arrangement and adjustment for training plans, judging whether fatigue occurs, effectively mining the potential of human body movement, improving the competitive ability and so on, and has become an important link of the scientific training [1].

\section{Analysis on Aerobic Capacity Index}

Aerobic capacity index consists of six types [2], respectively is "Maximal oxygen uptake, $\mathrm{VO}_{2} \max$ pleteau duration, Maximal lactate steady state, PWC170, Anaerobic threshold, Respiratory quotient". Among them, "Maximal oxygen uptake" is divided into "Direct Maximal oxygen uptake, Relative Maximal oxygen uptake"; "Anaerobic threshold" is divided into "Lactate Anaerobic threshold, Ventilation Anaerobic threshold, Break point Anaerobic threshold". The index system structure is shown in Fig. 1.

\section{Maximal oxygen uptake}

Maximal oxygen uptake $\left(\mathrm{VO}_{2} \mathrm{max}\right)$ refers to the amount of oxygen that can be ingested in the unit time when cardio-pulmonary function and the capacity muscle uses oxygen reach the limit level during the human body is doing the strenuous exercise with a long period of time which a large number of muscle groups participate in. [3]. Representation methods have two kinds of 
absolute value and relative value. Absolute value refers to the maximum amount of oxygen that the body can absorb in a unit of time, usually with one $\mathrm{L} / \mathrm{min}$; relative value is the maximum oxygen uptake calculated by per kilogram of body weight, with $\mathrm{ml} / \mathrm{kg} / \mathrm{min}$ as the unit. The influencing factors of maximum oxygen uptake mainly include three aspects:

One is that oxygen transport system's influence on the $\mathrm{VO}_{2} \max$. Pulmonary ventilation and ventilation function is one of the factors affect the human body oxygen absorption ability. The content and capacity of carrying oxygen of hemoglobin are closely related to the $\mathrm{VO}_{2} \mathrm{max}$; the capacity that blood moves oxygen is determined the transport efficiency of the cycle system in the unit time, which is restricted by stroke volume and heart rate. Therefore, the difference of cardiac output that the people with training and without training deal with maximum load work is mainly caused by the stroke volume.

Second is that the effect of the ability that muscle tissue makes use of oxygen on the $\mathrm{VO}_{2}$ max. The ability that muscle tissue uses oxygen is mainly related to the muscle fiber types and its metabolic characteristics. Slow-twitch muscle fibers have abundant capillary distribution, the number of mitochondria in muscle fibers is large, large volume and oxidase activity is high, myoglobin content is also higher. These characteristics of chronic fibers are beneficial to increase the oxygen uptake capacity of slow-twitch muscle fibers.

Third is the influence of other factors on the $\mathrm{VO}_{2} \max$. Genetic factors, there is a close relationship between the $\mathrm{VO}_{2} \max$ and genetic inherit, the possibility that training improves the $\mathrm{VO}_{2}$ max is smaller; age and gender factors, the $\mathrm{VO}_{2}$ max increases with age growth in the children's time, and gender differences appear in adolescence ; training factors, in the process that training causes the $\mathrm{VO}_{2}$ max increase, the early increase mainly depends on the enlargement of cardiac output, the late increase mainly depends on the enlargement of the ability that muscular tissue makes use of oxygen.

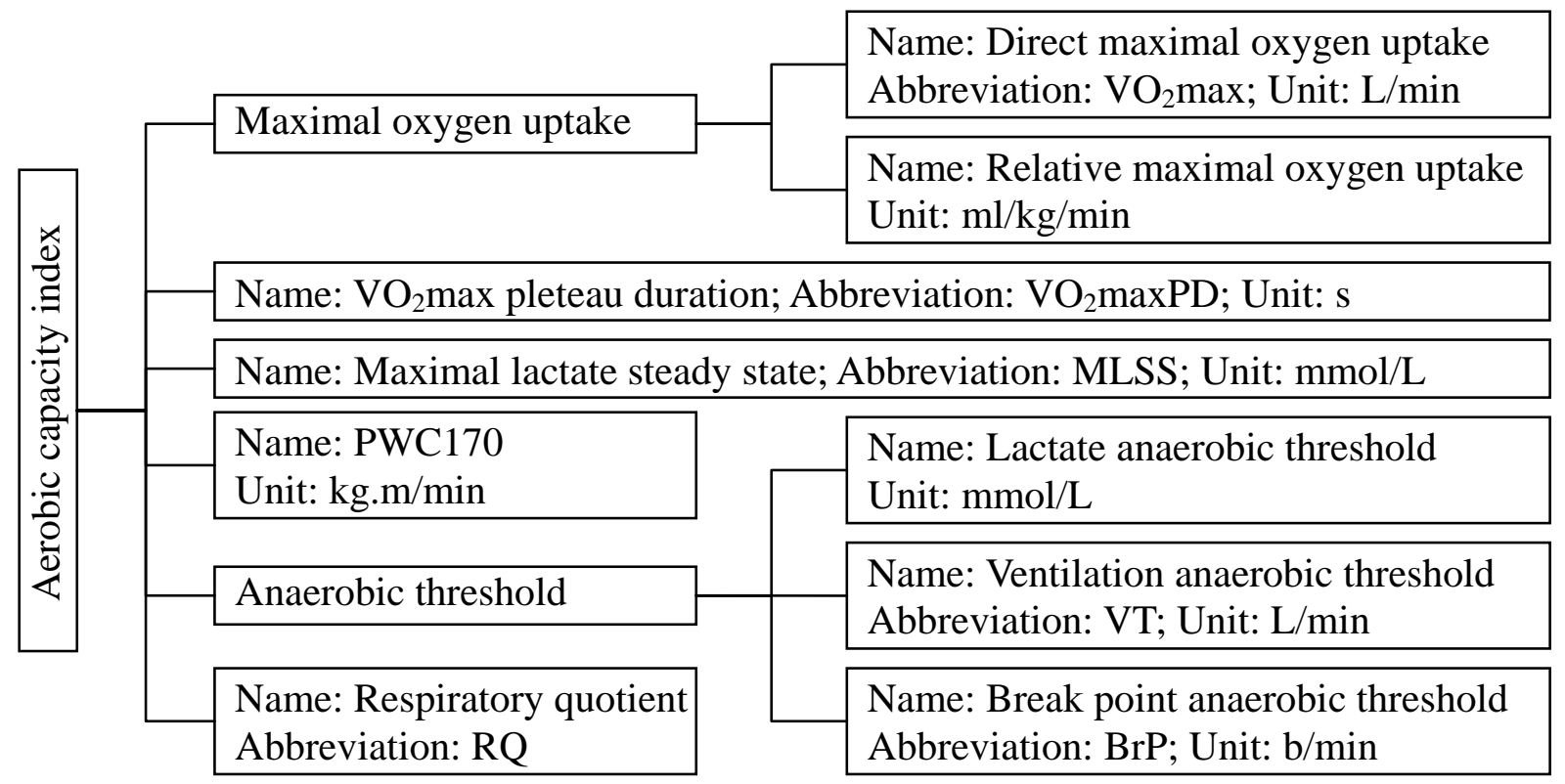

Fig. 1. Index system structure on aerobic capacity

\section{2. $\mathrm{VO}_{2}$ max pleteau duration}

$\mathrm{VO}_{2}$ max pleteau duration ( $\left.\mathrm{VO}_{2} \mathrm{maxPD}\right)$ is the continuous time to maintain the incremental intensity exercise process in the $\mathrm{VO}_{2}$ max level, and it proves that $\mathrm{VO}_{2}$ maxPD is significantly related with the aerobic exercise capacity by experiments [4]. Like $\mathrm{VO}_{2}$ max, heart and lung function is the most important physiological mechanism to restrict $\mathrm{VO}_{2}$ maxPD, which is determined mainly by the heart pump blood function, hemodynamics and the ability that blood carries oxygen. Peripheral muscle metabolism ability is also related with the duration of the platform, depending on the difference of arteriovenous oxygen and cells respiratory function, which has a close relationship with the muscle capillary network opening quantity, related enzyme activity, 
mitochondrial calcium metabolism ability, skeletal muscle fiber type, muscle acid ability, the ability that muscle removes lactic acid.

\section{Maximal lactate steady state}

Maximal lactate steady state refers to the maximum running speed or maximum power that the generation and elimination of lactic acid reach balance in the continuous constant load movement. MLSS is of great significance in physiology, which limits the critical intensity that a large number of anaerobic metabolism participate in supplying energy with the increase of demands that movements on energy. Analysis from the angle of the exercise intensity, when exercise intensity is less than or equal to the MLSS, the body lactic acid generation rate and removal rate are equal, both reach the balanced state; when exercise intensity is higher than the MLSS, lactic acid production rate is higher than removal rate, lactic acid in the body rises and there will be an inflection point of BLa-p or BLa-t curve. MLSS is a physiological phenomenon, which can be used as an indicator to monitor the training intensity $[5,6]$.

\section{PWC170}

PWC170 refers to determine the work done by the body in the unit time when the body function is mobilized and in a relatively stable state, heart rate is one hundred and seventy times per minute in the situation that the body is doing quantitative load exercise, which reflects the body's working capacity especially the aerobic endurance level. WC170 determination belongs to the sub limit quantitative load exercise test. Direct measurement is relatively complex, takes longer, the method of indirect determination is usually adopted. The principle of indirect determination of PWC170 is: movement process center rate and power within a certain load range (equivalent to the heart rate within one hundred and twenty and eighty times per minute) show a linear relationship. According to this relationship, the subjects complete the different load movement twice or more than twice, the first load makes the heart rate reaches about one hundred and twenty times per minute, the second load makes heart rate one hundred and seventy times per minute as close as possible. Through two load powers and two heart rates after loading, the work done by the body when heart rate is one hundred and seventy times per minute can be calculated.

\section{Anaerobic threshold}

Anaerobic threshold refers to the critical point that aerobic metabolism begins to transform to the anaerobic metabolism when the human body is increasing load intensity, anaerobic threshold is expressed by the corresponding intensity when blood lactic acid concentration reaches four mmol/L, ventilation and heart rate. Anaerobic threshold expressed by lactic acid concentration is called lactic acid anaerobic threshold, which is expressed by the change of ventilation and gas exchange is called ventilation anaerobic threshold. Anaerobic threshold expressed by the heart rate turning point extracted by heart rate and stroke volume rising slope changes is called heart rate anaerobic threshold. The athletes' aerobic capacity is not only determined by the maximum oxygen uptake, at the same time, which has also a very close relationship with anaerobic threshold, because the maximum oxygen uptake only reflects the oxygen intake capacity, however, anaerobic threshold can more accurately reflect the ability that skeletal muscle uses oxygen.

Anaerobic threshold can be greatly improved through systematic training [7]. How to use the anaerobic threshold to control the training intensity and get the best training effect is the main research topic in the current sports training. Because anaerobic threshold intensity training can make all kinds of motor function activities reach or close to the limit level, the proportion of anaerobic metabolism and energy metabolism reduced to a minimum, this is the physiological theory that anaerobic threshold theory makes training load optimization. Consideration from physiological load intensity, in order to improve the anaerobic threshold, coaches' training intensity is often used. Anaerobic threshold is the most commonly used index to evaluate athletes' aerobic metabolism abilities, when coaches arrange training plans, the training core is to fully improve athlete's anaerobic threshold speed. 


\section{Respiratory quotient}

Respiratory quotient (RQ) is also known as gas exchange rate, which refers to the ratio of the volume or moles that organisms at the same time release carbon dioxide and absorb oxygen, namely the molecular ratio that released $\mathrm{CO}_{2}$ and absorbed $\mathrm{O}_{2}$ by the respiratory function. Due to the different energy order of sugar, lipid and protein, only when sugar and lipid energy are shortage, protein oxidation power can appear. This situation rarely occurs in organisms, so usually the measured respiratory quotient is in fact the non-protein respiratory quotient, that is the respiratory quotient measured by sugar and lipid as the substrate of oxidation decomposition.

\section{Design on Information Management Data Structure}

Data structure is organized by the data elements according to a certain logic connection. The description of the logical relationship between the data and elements is called the logical structure of data; data must be stored in the computer, the data storage structure is the realization form of data structure, the representation in the computer; a data structure must be performed on the data operation at the same time to be meaningful. A logical data structure can have a variety of storage structures, and all kinds of storage structures affect the efficiency of data processing. The choice of data structure is a basic design consideration factor. The construction experience of many large-scale systems shows that the difficult level of the system implementation and the quality of the system construction are all heavily dependent on whether choose the optimal data structure. In the software system, data is usually stored in the database, this article designs data structure based on MySQL database management system, shown in Table 1.

In the above three tables, involving three class and six types of data types.

First, string data types, the most commonly used data types, respectively for fixed-length and variable-length strings. Fixed-length string is a fixed length character, the length is designated when creating a table, not allowed more than the specified character data, the allocated storage space is in accordance with specified, this system selects the Char type. Variable-length string is divided into two kinds, one kind is the largest fixed-length, this system chooses the Varchar type; one is complete variable length, this system chooses the Text type [8].

Secondly, numeric data types, used to store value, each type has a different range of storage, the greater support scope is, the more storage space requires. Data types are divided into integer and floating point, the system integer storage data range is smaller, the Tinyint type is selected; floating point type requires a higher precision, the Decimal type is chose.

Thirdly, date and time data types. There are a variety of data types represent the data and time in MySQL. Year represents the Year, Date represents the date, Time represents the time, Datetime and Timestamp indicate the date and time. The system date and time data types are divided into two categories, the one data type only needs to save the date, namely "yyyy-mm-dd", chooses the Date data type; another data type needs to save date and time, namely "yyyy-mm-dd hh:mm:ss", chooses the Datetime data type.

\section{Conclusion}

Aerobics exercise has a lot of exercise forms and programs, such as brisk walking, jogging, riding a bicycle, jumping rope, dancing fitness dance, skating, swimming, playing ball, playing tai chi, climbing stairs, boating and so on. [9]. Studies have indicated that the key of taking aerobic metabolism exercise is to guarantee a certain amount of exercise and persistence. Adults selectively participate in the sports programs to exercise can achieve the effect of moderate aerobic exercise. From the point of the view of athletes' various aerobic training methods at present, grasping the exercise maximum intensity is not enough, the phenomenon that aerobic exercise intensity is too high is widespread; special aerobic training method is relatively unitary, monotonous forms, boring contents, which cannot inspire athletes' training enthusiasm, lack of combining with special game technical aerobic training methods. The research results of this paper are of great significance for the people's health, high level athletes training and information construction. 
Table 1. Athlete information table

\begin{tabular}{c|c|c|c|l}
\hline No & Name & Fields Type & Fields Width & \multicolumn{1}{|l}{ Notes and Instructions } \\
\hline 1 & YDYBH & Varchar & 10 & Athlete number \\
\hline 2 & YDYXM & Varchar & 30 & Athlete name \\
\hline 3 & YDYXB & Char & 2 & Athlete sex \\
\hline 4 & YDYCSRQ & Date & 4 & Athlete date of birth \\
\hline 5 & YDXXMC & Varchar & 30 & Sport item name \\
\hline 6 & RDRQSJ & Date & 4 & In team date \\
\hline 7 & LXDH & Varchar & 50 & Contact phone \\
\hline 8 & DXYX & Varchar & 50 & Electronic mail \\
\hline 9 & TXDZ & Varchar & 100 & Mailing address \\
\hline 10 & YZBM & Char & 6 & Postcode \\
\hline 11 & YDJL & Text & 8 & Sports resume \\
\hline
\end{tabular}

Table 2. Sampling information table

\begin{tabular}{c|c|c|c|l}
\hline No & Name & Fields Type & Fields Width & \multicolumn{1}{|l}{ Notes and Instructions } \\
\hline 21 & YDYBH & Varchar & 10 & Athlete number \\
\hline 22 & CYBH & Varchar & 10 & Sampling number \\
\hline 23 & CYRQSH & Datetime & 8 & Sampling date time \\
\hline 24 & CYDD & Varchar & 100 & Sampling sites \\
\hline 25 & CYRY & Varchar & 30 & Sampling personnel \\
\hline 26 & CYWD & Decimal & 5,2 & Sampling temperature \\
\hline 27 & HJZK & Varchar & 500 & Sampling environmental status \\
\hline 28 & CYQKSM & Text & 8 & Sampling situation instructions \\
\hline
\end{tabular}

Table 3. Index value information table

\begin{tabular}{c|c|c|c|l}
\hline No & Name & Fields Type & Fields Width & \multicolumn{1}{|c}{ Notes and Instructions } \\
\hline 31 & YDYBH & Varchar & 10 & Athlete number \\
\hline 32 & CYBH & Varchar & 10 & Sampling number \\
\hline 33 & CLJGRQSH & Datetime & 8 & Measuring result date time \\
\hline 34 & ZDSYL & Decimal & 10,4 & Direct maximal oxygen uptake \\
\hline 35 & XDZDSYL & Decimal & 10,4 & Relative maximal oxygen uptake \\
\hline 36 & ZDSYLPT & Decimal & 10,4 & VO \\
\hline 37 & PWC & Decimal & 10,4 & PWC170 pleteau duration \\
\hline 38 & MDRSWT & Decimal & 10,4 & Maximal lactate steady state \\
\hline 39 & RSWYY & Decimal & 10,4 & Lactate anaerobic threshold \\
\hline 40 & TQWYY & Decimal & 10,4 & Ventilation anaerobic threshold \\
\hline 41 & XLWYY & Decimal & 10,4 & Break point anaerobic threshold \\
\hline 42 & HXS & Decimal & 10,4 & Respiratory quotient \\
\hline 43 & BZSM & Text & 8 & Notes and Instructions \\
\hline
\end{tabular}




\section{Acknowledgement}

This work is supported by general subject of "the Twelfth Five-year Plan" on educational science of Liaoning province: Action Research on Sensory Integration Training Integrate into Children's Sports Activities, 2015, Approval (JG15CB167).

\section{References}

[1] K. B. Jia, "On Evaluation of aerobic metabolism ability of athletes," The Science Education Article Collects, vol. 3, no. 6, pp. 279, 2009.

[2] General Administration of Sport of China, "Requirements of athletes' physical morphometrics and functions digital archives," Sports industry standard of the people's Republic of China, 2014-5-22.

[3] Douban net, "maximal oxygen uptake," http://www.douban.com/note/486430042/, 2016-1-11.

[4] Q. Shen, Y. P. Zhou, A. P. Lai, "A Review of the Measurements and Application of Maximum Oxygen Uptake Plateau Duration," Zhejiang Sport Science, vol. 28, no. 1, pp. 91-93, 2006.

[5] Z. R. Xia, "Assessment of maximal lactate steady state response in race walking," Master's Degree of Beijing Sport University, 2011.

[6] Z. Su, "Devel system," Experimental Research on Aerobic Capacity of Man Sanda Athletes, vol. 34, no. 5, pp. 81-83, 2013.

[7] T. H. Tian, J. Li, "Application of lactate anaerobic threshold in marathon project," Intelligence, vol. 14, no. 9, pp. 352-353, 2014.

[8] L. Huang, D. Y. Liu, "Research on the Public Bicycle Service System by MySQL," Journal of Hubei University for Nationalities (Natural Science Edition), vol. 32, no. 1, pp. 78-80, 2014.

[9] Baidu Encyclopedia, "Aerobic metabolism," http://baike.baidu.com/link?url=6SzbzDM7g4e30ATSp8OL_AeKUtuP4HwWDdVLqHZ5B6H _cDLCrBzVaaT0kqnHU5Kqj71choB7JhoL9-AfP1cSbq, 2016-1-11. 